\title{
Properties of noncommutative axionic electrodynamics
}

\author{
Patricio Gaete* and Iván Schmidt田 \\ Center of Subatomic Studies and Departmento de Física \\ Universidad Técnica Federico Santa María, Valparaíso, Chile
}

(Dated: August 9, 2021)

\begin{abstract}
Using the gauge-invariant but path-dependent variables formalism, we compute the static quantum potential for noncommutative axionic electrodynamics, and find a radically different result than the corresponding commutative case. We explicitly show that the static potential profile is analogous to that encountered in both non-Abelian axionic electrodynamics and in Yang-Mills theory with spontaneous symmetry breaking of scale symmetry.

PACS numbers: 11.10.Ef, 11.15.-q
\end{abstract}

The coupling of axion-like particles (or "axions") with photons in the presence of an external background electromagnetic field and its physical consequences such as vacuum birefringence and vacuum dichroism have been the object of intensive investigations by many authors [1, 2, 3, 4, 5, 6, 7, 8, 9]. Moreover, as is well known, this subject has received increasing attention after recent results of the PVLAS collaboration [10], in which dichroism [10] and birefringence [11] were observed for a linearly polarized laser beam after it traverses an external magnetic field. We recall that these effects can be qualitatively understood by the existence of light pseudoscalars bosons $\phi$ ("axions"), with a coupling to two photons. In this case, the corresponding term in the effective Lagrangian has the form $\mathcal{L}_{I}=-1 / 4 M F_{\mu \nu} \widetilde{F}^{\mu \nu} \phi$, where $\widetilde{F}^{\mu \nu}=1 / 2^{\varepsilon_{\mu \nu \lambda \rho}} F^{\lambda \rho}$. Certainly, if the PVLAS results are confirmed by further experimental data, it would signal new physics containing very light bosons [12]. Meanwhile, alternatives models have been suggested in order to circumvent the discrepancy among the results of the PVLAS experiment with both astrophysical bounds and the results of the CAST collaboration [6, 13]. For example, in Ref. [13] the existence of a new light vector field ( rather than an axion field) was considered, which interacts with the photon via Chern-Simons-like terms. Another possible scenario is the existence of millicharged particles [14]. In addition, the study of spacetime non-commutativity on light propagation in a background electromagnetic field has also attracted interest in order to account the results reported by PVLAS collaboration [15, 16]. In particular, the non-commutative axionic electrodynamics version has been considered [16] within the framework of the Lorentz violating extension of QED [17.

On the other hand, axionic electrodynamics experiences mass generation due to the breaking of rotational invariance induced by a classical background configuration of the gauge field strength [18], and leads to confining potentials in the presence of nontrivial constant expecta-

\footnotetext{
*Electronic address: patricio.gaete@usm.cl
}

†Electronic address: ivan.schmidt@usm.cl tion values for the gauge field strength $F_{\mu \nu}$ [19]. In fact, in the case of a constant electric field strength expectation value the static potential remains Coulombic, while in the case of a constant magnetic field strength expectation value the potential energy is the sum of a Yukawa and a linear potential, leading to the confinement of static charges. In passing we note that the distinction between the apparently related phenomena of screening and confinement is of considerable importance in our present understanding of gauge theories. We also mention that the magnetic character of the field strength expectation value needed to obtain confinement is in agreement with the current chromo-magnetic picture of the $Q C D$ vacuum [20]. Interestingly, similar results have been obtained in the context of the dual Ginzburg-Landau theory [21], as well as for a theory of antisymmetric tensor fields that results from the condensation of topological defects as a consequence of the Julia-Toulouse mechanism 22]. It must be clear from this discussion that the interaction potential between static charges is an object of utmost importance, which has a variety of applications. For instance, it provides a useful framework for studying aspects of duality in Maxwell-like three-dimensional models induced by the condensation of topological defects driven by quantum fluctuations [23]. Also, it has proven to be a key tool to analyze the equivalence/nonequivalence between the $\theta$-expanded version of the noncommutative $\mathrm{U}(1)$ gauge theory and the Born-Infeld action up to order $F^{3}[24]$.

Inspired by these observations, the purpose of this note is to further elaborate on the physical content of noncommutative axionic electrodynamics [16]. We examine another aspect of this theory, namely, the effect of the space-time noncommutativity on a physical observable. To do this, we will work out the static potential for the theory under consideration by using the gaugeinvariant but path-dependent variables formalism, which is alternative to the Wilson loop approach. Our treatment is fully non-perturbative for the pseudoscalar field. As a result, we obtain a Cornell-like potential which displays a marked departure of a qualitative nature from the results of the commutative case [19] at large distances, and which clearly shows the key role played by the noncommutative space in transforming the Yukawa 
potential into the Coulombic one. Incidentally, the above static potential profile is analogous to that encountered in both non-Abelian axionic electrodynamics [25] and in Yang-Mills theory with spontaneous symmetry breaking of scale symmetry [26]. In this way we establish a new connection between noncommutative and non-Abelian effective theories. The above connections are of interest from the point of view of providing unifications among diverse models as well as exploiting the equivalence in explicit calculations, as we are going to show. Moreover, our work could be of interest for searching for small violations of the Lorentz symmetry along the lines of Ref. [27].

We now examine the interaction energy between static point-like sources for noncommutative axionic electrodynamics. To do this, we will compute the expectation value of the energy operator $H$ in the physical state $|\Phi\rangle$ describing the sources, which we will denote by $\langle H\rangle_{\Phi}$. However, before going to the derivation of the interaction energy, we will describe very briefly the model under consideration. The initial point of our analysis is the Lagrangian density:

$$
\mathcal{L}=\mathcal{L}_{L V-Q E D}+\mathcal{L}_{\text {Axion }}
$$

where $\mathcal{L}_{L V-Q E D}=-\frac{1}{4} F_{\mu \nu} F^{\mu \nu}-\frac{1}{4}\left(k_{F}\right)_{\mu \nu \rho \sigma} F^{\mu \nu} F^{\rho \sigma}+$ $\frac{1}{2}\left(k_{A F}\right)^{\mu} \varepsilon_{\mu \nu \rho \sigma} A^{\nu} F^{\rho \sigma}$ represents the Lorentz-violating extension of QED [17], and $\mathcal{L}_{\text {Axion }}=\frac{1}{2}(\partial \phi)^{2}-\frac{m^{2}}{2} \phi^{2}+$ $\frac{\phi}{8 M} \varepsilon_{\mu \nu \rho \sigma} F^{\mu \nu} F^{\rho \sigma}$ describes the coupling of a pseudoscalar $\phi$ to electromagnetism. Next, the corresponding noncommutative version of (1) is induced by the Moyal product $f * g=f \exp \left(\frac{i}{2} \overleftarrow{\partial}_{\mu} \theta^{\mu \nu} \vec{\partial}_{\nu}\right) g$, where $\theta^{\mu \nu}$ is a real constant antisymmetric tensor. As a consequence, the field strength $\widehat{F}_{\mu \nu}$ in a noncommutative space-time is given by $\widehat{F}_{\mu \nu}=\partial_{\mu} \widehat{A}_{\nu}-\partial_{\nu} \widehat{A}_{\mu}-i g\left[\widehat{A}_{\mu}, \widehat{A}_{\nu}\right]$ and the bracket denotes a Moyal commutator. This then implies that the theory is invariant under a non conventional gauge transformation $\delta \widehat{A}_{\mu}=\partial_{\mu} \widehat{\lambda}-i g\left[\widehat{A}_{\mu}, \widehat{\lambda}\right]$. Now we can exploit the Seiberg-Witten map $\widehat{A}_{\mu}=A_{\mu}-\frac{1}{2} \theta^{\alpha \beta} A_{\alpha}\left(\partial_{\beta} A_{\mu}+F_{\beta \mu}\right)$, to get a gauge field $A_{\mu}$ with the ordinary gauge transformation and a Lagrangian density written in terms of the conventional field strength. Accordingly, after splitting $F_{\mu \nu}$ in the sum of a classical background $\left\langle F_{\mu \nu}\right\rangle$ and a small fluctuation $f_{\mu \nu}=\partial_{\mu} A_{\nu}-\partial_{\nu} A_{\mu}$, the corresponding Lagrangian density to leading order in $\theta$ ( with $\theta^{0 i}=0$ and $\left.\left(k_{A F}\right)^{\mu}=0\right)$ is given by [16]:

$$
\begin{aligned}
\mathcal{L} & =-\frac{1}{4}\left(1-\frac{g}{2} \theta^{i j}\left\langle F_{i j}\right\rangle\right) f^{\mu \nu} f_{\mu \nu}+\frac{g}{4} \theta^{i j} f_{i j}\left\langle F_{k l}\right\rangle f^{k l} \\
& -\frac{g}{2} \theta_{i j}\left\langle F_{k l}\right\rangle f^{k i} f^{l j}-g \theta_{i j}\left\langle F^{k i}\right\rangle f_{\mu k} f^{j \mu}+\frac{1}{2}(\partial \phi)^{2} \\
& -\frac{m^{2}}{2} \phi^{2}+\frac{1}{4 M} \varepsilon^{\rho \sigma \mu \nu}\left\langle F_{\rho \sigma}\right\rangle f_{\mu \nu} \phi
\end{aligned}
$$

Throughout, $M$ and $g$ are the photon-axion and $\mathrm{U}(1)$ gauge coupling respectively. To get the last equation we used $\left(k_{F}\right)_{\mu \nu \rho \sigma}=\frac{1}{8}\left(T_{[\mu \nu][\rho \sigma]}+\mu \nu \leftrightarrow \rho \sigma\right)$, where $T_{\mu \nu \rho \sigma} \equiv-\frac{1}{2} g \theta^{\alpha \beta}\left\langle F_{\alpha \beta}\right\rangle \eta_{\mu \rho} \eta_{\nu \sigma}-g \theta_{\mu \nu}\left\langle F_{\rho \sigma}\right\rangle+$ $4 g \theta_{\alpha \nu}\left\langle F_{\rho}^{\alpha}\right\rangle+2 g \theta_{\nu \sigma}\langle F\rangle_{\mu \rho}$, and $T_{[\mu \nu]} \equiv T_{\mu \nu}-T_{\nu \mu}$. Here $(\mu, \nu, \rho, \sigma=0,1,2,3)$ and $(i, j, l, k=1,2,3)$.

Following our earlier procedure [19], integrating out the $\phi$ field induces an effective theory for the $A_{\mu}$ field. Once this is done, we arrive at the following effective Lagrangian density:

$$
\begin{aligned}
\mathcal{L} & =-\frac{1}{4}\left(1-\frac{g}{2} \theta^{i j}\left\langle F_{i j}\right\rangle\right) f^{\mu \nu} f_{\mu \nu}+\frac{g}{4} \theta^{i j} f_{i j}\left\langle F_{k l}\right\rangle f^{k l} \\
& -\frac{g}{2} \theta_{i j}\left\langle F_{k l}\right\rangle f^{k i} f^{l j}-g \theta_{i j}\left\langle F^{k i}\right\rangle f_{\mu k} f^{j \mu} \\
& +\frac{1}{32 M^{2}} v^{\mu \nu} f_{\mu \nu} \frac{1}{\Delta+m^{2}} v^{\gamma \delta} f_{\gamma \delta}
\end{aligned}
$$

Here we have simplified our notation by setting $\varepsilon^{\mu \nu \alpha \beta}\left\langle F_{\mu \nu}\right\rangle \equiv v^{\alpha \beta}$ and $\varepsilon^{\rho \sigma \gamma \delta}\left\langle F_{\rho \sigma}\right\rangle \equiv v^{\gamma \delta}$. One immediately sees that this expression has a remarkable similarity to the corresponding commutative effective Lagrangian density. This common feature is our main motivation for studying the role of the noncommutative space on the interaction energy. With this in view, we now proceed to calculate the interaction energy in the $v^{0 i} \neq 0$ and $v^{i j}=0$ case (referred to as the magnetic one in what follows). Using this in (3) we then obtain

$$
\begin{aligned}
\mathcal{L} & =-\frac{1}{4}\left(1-\frac{g}{2} \theta^{i j}\left\langle F_{i j}\right\rangle\right) f^{\mu \nu} f_{\mu \nu}+\frac{g}{4} \theta^{i j} f_{i j}\left\langle F_{k l}\right\rangle f^{k l} \\
& -\frac{g}{2} \theta_{i j}\left\langle F_{k l}\right\rangle f^{k i} f^{l j}-g \theta_{i j}\left\langle F^{k i}\right\rangle f_{\mu k} f^{j \mu} \\
& +\frac{1}{16 M^{2}} v^{0 i} f_{0 i} \frac{1}{\Delta+m^{2}} v^{0 k} f_{0 k}
\end{aligned}
$$

We observe that the limit $\theta \rightarrow 0$ is well defined, and leads to the corresponding commutative Lagrangian density. To obtain the corresponding Hamiltonian, we must carry out the quantization of this theory. The Hamiltonian analysis starts with the computation of the canonical momenta $\Pi^{\mu}=-\left(1-\frac{g}{2} \theta^{i j}\left\langle F_{i j}\right\rangle\right) f^{0 \mu}-$ $g \theta_{i j}\left\langle F^{\mu i}\right\rangle f^{j 0}+g \theta^{i \mu}\left\langle F^{k i}\right\rangle f^{0 k}+\frac{1}{16 M^{2}} v^{0 \mu} \frac{1}{\Delta+m^{2}} v^{o i} f_{0 i}$, which produces the usual primary constraint $\Pi^{0}=0$ and $\Pi_{i}=\left(1-\frac{g}{2} \theta^{i j}\left\langle F_{i j}\right\rangle\right) f_{i 0}-g\left[\theta_{k j}\left\langle F_{k j}\right\rangle+\theta_{k i}\left\langle F_{j k}\right\rangle\right] f_{j 0}+$ $\frac{1}{16 M^{2}} v_{i 0} \frac{1}{\Delta+m^{2}} v_{j 0} f_{j 0}$. The canonical Hamiltonian to leading order in $\theta$ and $\frac{1}{M^{2}}$ is then

$$
\begin{aligned}
H_{C} & =\int d^{3} x\left\{-A^{0}\left(\partial_{i} \Pi^{i}\right)+\frac{[1+g(\theta \cdot \mathcal{B})] \mathbf{\Pi}^{2}}{2}\right\} \\
& +\int d^{3} x\left\{\frac{(\theta \cdot \mathcal{B})}{2} \mathbf{B}^{2}-\frac{g(\theta \cdot \mathbf{B})}{2} \mathcal{B} \cdot \mathbf{B}\right\} \\
& +\int d^{3} x\left\{g \theta_{i j}\left[\frac{\left\langle F_{k l}\right\rangle}{2} f^{k i}-\left\langle F^{k i}\right\rangle f_{l k}\right] f^{l j}\right\} \\
& +\int d^{3} x \frac{g(\mathcal{B})^{2}(\theta \cdot \mathcal{B})}{32 M^{2}} \Pi^{i}\left(\Delta+m^{2}\right)^{-1} \Pi^{i} \\
& -\int d^{3} x \frac{(\mathcal{B})^{2}[1+5 g(\theta \cdot \mathcal{B})]}{32 M^{2}} \Pi^{i}\left(\Delta+\mathcal{M}^{2}\right)^{-1} \Pi^{i},
\end{aligned}
$$


where $\mathcal{M}^{2} \equiv m^{2}+\frac{\mathbf{v}^{2}}{16 M^{2}}=m^{2}+\frac{\mathcal{B}^{2}}{4 M^{2}}$. Here $\mathbf{B}$ and $\mathcal{B}$ represent the magnetic field and external magnetic field, respectively. Requiring the primary constraint $\Pi_{0}=0$ to be preserved in time yields the secondary constraint $\Gamma_{1}(x) \equiv \partial_{i} \Pi^{i}=0$. But the time stability of the secondary constraint does not induce further constraints. Therefore, the extended Hamiltonian that generates translations in time then reads $H=$ $H_{C}+\int d^{3} x\left(c_{0}(x) \Pi_{0}(x)+c_{1}(x) \Gamma_{1}(x)\right)$, where $c_{0}(x)$ and $c_{1}(x)$ are the Lagrange multiplier fields. Moreover, it is straightforward to see that $\dot{A}_{0}(x)=\left[A_{0}(x), H\right]=$ $c_{0}(x)$, which is an arbitrary function. Since $\Pi^{0}=0$ always, neither $A^{0}$ nor $\Pi^{0}$ are of interest in describing the system and may be discarded from the theory. Thus the Hamiltonian is now given as

$$
\begin{aligned}
H_{C} & =\int d^{3} x\left\{c(x)\left(\partial_{i} \Pi^{i}\right)+\frac{[1+g(\theta \cdot \mathcal{B})] \mathbf{\Pi}^{2}}{2}\right\} \\
& +\int d^{3} x\left\{\frac{(\theta \cdot \mathcal{B})}{2} \mathbf{B}^{2}-\frac{g(\theta \cdot \mathbf{B})}{2} \mathcal{B} \cdot \mathbf{B}\right\} \\
& +\int d^{3} x\left\{g \theta_{i j}\left[\frac{\left\langle F_{k l}\right\rangle}{2} f^{k i}-\left\langle F^{k i}\right\rangle f_{l k}\right] f^{l j}\right\} \\
& +\int d^{3} x \frac{g(\mathcal{B})^{2}(\theta \cdot \mathcal{B})}{32 M^{2}} \Pi^{i}\left(\Delta+m^{2}\right)^{-1} \Pi^{i} \\
& -\int d^{3} x \frac{(\mathcal{B})^{2}[1+5 g(\theta \cdot \mathcal{B})]}{32 M^{2}} \Pi^{i}\left(\Delta+\mathcal{M}^{2}\right)^{-1} \Pi^{i}
\end{aligned}
$$

where $c(x)=c_{1}(x)-A_{0}(x)$.

In accordance with the Dirac method, we must fix the gauge. A particularly convenient gauge fixing condition is

$$
\Gamma_{2}(x) \equiv \int_{C_{\xi x}} d z^{\nu} A_{\nu}(z) \equiv \int_{0}^{1} d \lambda x^{i} A_{i}(\lambda x)=0,
$$

where $\lambda(0 \leq \lambda \leq 1)$ is the parameter describing the spacelike straight path $x^{i}=\xi^{i}+\lambda(x-\xi)^{i}$, and $\xi$ is a fixed point (reference point). There is no essential loss of generality if we restrict our considerations to $\xi^{i}=0$. The choice (7) leads to the Poincaré gauge [28, 29]. Through this procedure, we arrive at the only nonvanishing equaltime Dirac bracket for the canonical variables

$$
\begin{aligned}
\left\{A_{i}(x), \Pi^{j}(y)\right\}^{*} & =\delta_{i}^{j} \delta^{(3)}(x-y) \\
& -\partial_{i}^{x} \int_{0}^{1} d \lambda x^{j} \delta^{(3)}(\lambda x-y) .
\end{aligned}
$$

After achieving the quantization we may now proceed to calculate the interaction energy in the model under consideration. As mentioned above, we will work out the expectation value of the energy operator $H$ in the physical state $|\Phi\rangle$. Now we recall that the physical states $|\Phi\rangle$ are gauge-invariant [30]. In that case we consider the stringy gauge-invariant state

$$
\begin{aligned}
|\Phi\rangle & \equiv|\bar{\Psi}(\mathbf{y}) \Psi(\mathbf{y} \prime)\rangle \\
& =\bar{\psi}(\mathbf{y}) \exp \left(i q \int_{\mathbf{y}^{\prime}}^{\mathbf{y}} d z^{i} A_{i}(z)\right) \psi\left(\mathbf{y}^{\prime}\right)|0\rangle,
\end{aligned}
$$

where $|0\rangle$ is the physical vacuum state and the line integral appearing in the above expression is along a spacelike path starting at $\mathbf{y}^{\prime}$ and ending at $\mathbf{y}$, on a fixed time slice. The charged matter field together with the electromagnetic cloud (dressing) which surrounds it, is given by $\Psi(\mathbf{y})=\exp \left(-i q \int_{C_{\xi \mathbf{y}}} d z^{\mu} A_{\mu}(z)\right) \psi(\mathbf{y})$. Thanks to our path choice, this physical fermion then becomes $\Psi(\mathbf{y})=\exp \left(-i q \int_{\mathbf{0}}^{\mathbf{y}} d z^{i} A_{i}(z)\right) \psi(\mathbf{y})$. In other terms, each of the states $(|\Phi\rangle)$ represents a fermion-antifermion pair surrounded by a cloud of gauge fields to maintain gauge invariance.

From the foregoing Hamiltonian structure we then easily verify that

$$
\begin{aligned}
\Pi_{i}(x)\left|\bar{\Psi}(\mathbf{y}) \Psi\left(\mathbf{y}^{\prime}\right)\right\rangle & =\bar{\Psi}(\mathbf{y}) \Psi\left(\mathbf{y}^{\prime}\right) \Pi_{i}(x)|0\rangle \\
& +q \int_{\mathbf{y}}^{\mathbf{y}^{\prime}} d z_{i} \delta^{(3)}(\mathbf{z}-\mathbf{x})|\Phi\rangle
\end{aligned}
$$

Having made this observation and since the fermions are taken to be infinitely massive (static) we can substitute $\Delta$ by $-\nabla^{2}$ in Eq. (6). In such a case $\langle H\rangle_{\Phi}$ reduces to

$$
\langle H\rangle_{\Phi}=\langle H\rangle_{0}+\langle H\rangle_{\Phi}^{(1)}+\langle H\rangle_{\Phi}^{(2)}+\langle H\rangle_{\Phi}^{(3)},
$$

where $\langle H\rangle_{0}=\langle 0|H| 0\rangle$, and the $\langle H\rangle_{\Phi}^{(1)},\langle H\rangle_{\Phi}^{(2)}$ and $\langle H\rangle_{\Phi}^{(3)}$ terms are given by

$$
\langle H\rangle_{\Phi}^{(1)}=\alpha\left\langle\Phi\left|\int d^{3} x \Pi^{i} \Pi^{i}\right| \Phi\right\rangle,
$$

$$
\begin{gathered}
\langle H\rangle_{\Phi}^{(2)}=\beta\left\langle\Phi\left|\int d^{3} x \Pi^{i}\left(\nabla^{2}-m^{2}\right)^{-1} \Pi^{i}\right| \Phi\right\rangle, \\
\langle H\rangle_{\Phi}^{(3)}=\gamma\left\langle\Phi\left|\int d^{3} x \Pi^{i}\left(\nabla^{2}-\mathcal{M}^{2}\right)^{-1} \Pi^{i}\right| \Phi\right\rangle,
\end{gathered}
$$

with $\alpha \equiv \frac{[1+g(\theta \cdot \mathcal{B})]}{2}, \quad \beta \equiv \frac{g(\mathcal{B})^{2}(\theta \cdot \mathcal{B})}{32 M^{2}}$ and $\gamma \equiv$ $\frac{(\mathcal{B})^{2}[1+5 g(\theta \cdot \mathcal{B})]}{32 M^{2}}$. Using Eq. (10), the $\langle H\rangle_{\Phi}^{(1)},\langle H\rangle_{\Phi}^{(2)}$ and $\langle H\rangle_{\Phi}^{(3)}$ terms can be rewritten as

$$
\begin{aligned}
& \langle H\rangle_{\Phi}^{(1)}=q^{2} \alpha \int d^{3} x\left(\int_{\mathbf{y}^{\prime}}^{\mathbf{y}} \delta^{(3)}(\mathbf{x}-\mathbf{z})\right)^{2}, \\
& \langle H\rangle_{\Phi}^{(2)}=q^{2} \beta \int_{\mathbf{y}^{\prime}}^{\mathbf{y}} d z^{i} \int_{\mathbf{y}^{\prime}}^{\mathbf{y}} d z^{i} \frac{e^{-m\left|\mathbf{z}^{\prime}-\mathbf{z}\right|}}{4 \pi\left|\mathbf{z}^{\prime}-\mathbf{z}\right|},
\end{aligned}
$$




$$
\langle H\rangle_{\Phi}^{(3)}=q^{2} \beta \int_{\mathbf{y}^{\prime}}^{\mathbf{y}} d z^{\prime i} \int_{\mathbf{y}^{\prime}}^{\mathbf{y}} d z^{i} \frac{e^{-\mathcal{M}\left|\mathbf{z}^{\prime}-\mathbf{z}\right|}}{4 \pi\left|\mathbf{z}^{\prime}-\mathbf{z}\right|} .
$$

Following our earlier procedure [19, 25], we see that the potential for two opposite charges located at $\mathbf{y}$ and $\mathbf{y}^{\prime}$ takes the form

$$
V=-\frac{q^{2}[1+g(\theta \cdot \mathcal{B})]}{4 \pi} \frac{1}{L}+\frac{q^{2}(\mathcal{B})^{2}}{256 \pi M^{2}} \sigma L,
$$

where

$\sigma \equiv g(\theta \cdot \mathcal{B}) \ln \left(1+\frac{\Lambda^{2}}{m^{2}}\right)+[1+5 g(\theta \cdot \mathcal{B})] \ln \left(1+\frac{\bar{\Lambda}^{2}}{\mathcal{M}^{2}}\right)$

while $\Lambda$ and $\bar{\Lambda}$ are a cuttoff and $\left|\mathbf{y}-\mathbf{y}^{\prime}\right| \equiv L$. As already observed, the consistent limit $\theta \rightarrow 0$ has to be taken in Eq. (4) in order to recover the commutative result.

In summary, we have considered the confinement versus screening issue for noncommutative axionic electrodynamics, in the case when there is an external magnetic field. Interestingly, we have obtained a Cornell-like potential profile. As already expressed, similar forms of interaction potentials have been reported before in the context of non-Abelian axionic electrodynamics 25] and in Yang-Mills theory with spontaneous symmetry breaking of scale symmetry [26]. In this way we have provided a new connection among diverse effective models. The above analysis reveals the key role played by the noncommutative space in transforming the Yukawa potential into the Coulombic one. Also, a common feature of these models is that the rotational symmetry is restored in the resulting interaction energy.

Let us also mention here that the static potential profile of these models is similar to that encountered in a $\mathrm{U}(1)$ gauge theory that includes the contribution of all topologically nontrivial sectors of the theory [31]. In this sense these models may be considered as a physical realization of the topological nontrivial sectors studied in Ref. 31]. We conclude by noting that further investigation of the relation between our result and that encountered in Yang-Mills theory with spontaneous symmetry breaking of scale symmetry has to be performed, if our analysis represents an effective approach to non-Abelian gauge theories and especially quark confinement.

P. G. was partially supported by Fondecyt (Chile) grant 1050546.
[1] L. Maiani, R. Petronzio and E. Zavattini, Phys. Lett. B175, 359 (1986).

[2] P. Sikivie, Phys. Rev. Lett. 51, 1415 (1983).

[3] G. Raffelt and L. Stodolsky, Phys. Rev. D37, 67 (1992).

[4] J. A. Grifols, E. Masso and R. Toldra, Phys. Rev. Lett. 77, 2372 (1996).

[5] P. Jain, S. Panda and S. Sarala, Phys. Rev. D66, 085007 (2002).

[6] E. Masso and J. Redondo, JCAP 0509, 015 (2005); Phys. Rev. Lett. 97, 151802 (2006).

[7] D. Lai and J. Heyl, Phys. Rev. D74, 123003 (2006).

[8] A. Mirizzi, G. Raffelt and P. Serpico, [arXiv: astro-ph/07043044].

[9] A. Ringwald, [arXiv: hep-th/07043195].

[10] E. Zavattini et al. [PVLAS collaboration], Phys. Rev. Lett. 96, 110406 (2006).

[11] G. Cantatore for the PVLAS collaboration talk at IDM 2006, Island of Rhodes, Grece, 11-16th September, 2006.

[12] K. van Bibber and J. Rosenberg, Phys. Today 59 N8, 30 (2006).

[13] I. Antoniadis, A. Boyarsky and O. Ruchayskiy, [arXiv: hep-ph/0606306.

[14] H. Gies, J. Jaeckel and A. Ringwald, Phys. Rev. Lett. 97, 140402 (2006).

[15] M. Chaichian, M. M. Sheik-Jabbari and A. Tureanu, [arXiv: hep-ph/0511323].

[16] N. Chatillon and A. Pinzul, Nucl. Phys. B764, 95 (2007).

[17] V. A. Kostelecky and M. Mewes, Phys. Rev. D66, 056005
(2002).

[18] S. Ansoldi, E. I. Guendelman and E. Spallucci, JHEP 09, 044 (2003).

[19] P. Gaete and E. I. Guendelman, Mod. Phys. Lett. A20, 319 (2005).

[20] G. K. Savvidy, Phys. Lett. B71, 133 (1977); H. B. Nielsen and P. Olesen, Nucl. Phys. B160, 380 (1979); H. B. Nielsen and M. Ninomiya, Nucl. Phys. B156, 1 (1979).

[21] H. Suganuma, S. Sasaki and H. Toki, Nucl. Phys. B435, 207 (1995).

[22] P. Gaete and C. Wotzasek, Phys. Lett. B601, 108 (2004).

[23] P. Gaete and C. Wotzasek, Phys. Lett. B625, 365 (2005).

[24] P. Gaete and I. Schmidt, Int. J. Mod. Phys. A19, 3247 (2004).

[25] P. Gaete and E. Spallucci, J. Phys. A39, 6021 (2006).

[26] P. Gaete, E. I. Guendelman and E. Spallucci, Phys. Lett. B649, 218 (2007).

[27] P. Gaete and C. Wotzasek, Phys. Rev. D75, 057902 (2007).

[28] P. Gaete, Z. Phys. C76, 355 (1997).

[29] P. Gaete and I. Schmidt, Phys. Rev. D61, 125002 (2000); Phys. Rev. D64, 027702 (2001).

[30] P. A. M. Dirac, The Principles of Quantum Mechanics (Oxford University Press, Oxford, 1958); Can. J. Phys. 33, 650 (1955).

[31] K.-I. Kondo, Phys. Rev. D58, 085013 (1998). 